\title{
Automated Tissue Processing of Plant Samples for SEM, TEM, and ImmunoEM in a Multi-user Educational Facility
}

\author{
Missy L. Hazen, Ruth A. Haldeman, Gang Ning \\ Electron Microscopy Facility in the Huck Institutes of the Life Sciences, The Pennsylvania State \\ University, University Park, PA 16802, USA
}

Automated tissue processors are primarily designed for clinical and medical service EM laboratories to process large numbers of human tissue samples for conventional transmission electron-microscopic analyses, whereas they are often underutilized in multi-user facilities due to the small number of samples processed for a given experiment and the wide variety of protocols employed. Here we report using an automated EM tissue processor to process plant samples for scanning electron microscopy (SEM), conventional TEM, and immunoEM (iEM) in a multi-user educational EM facility.

Leaves and flower buds of Arabidopsis thaliana were fixed in stacked sample baskets and processed in a Boeckeler RMC 5160 Auto EM Processor at $4{ }^{\circ} \mathrm{C}$ with programmed agitation every 10 seconds. Briefly, for SEM the samples were fixed with $2.8 \%$ glutaraldehyde in $0.1 \mathrm{M}$ HEPES buffer (pH7.2) containing $0.02 \%$ Triton X-100, washed and post-fixed in 1\% osmium tetroxide in the same buffer. After washing again with the above buffer, the samples were dehydrated through a graded series of ethanol prior to critical point drying, mounting and coating. For immunoelectron microscopy purposes, the samples were fixed with $4 \%$ paraformaldehyde and $0.5 \%$ glutaraldehyde in $0.1 \mathrm{M}$ HEPES buffer ( $\mathrm{pH} 7.2$ ) containing $0.02 \%$ Triton X-100, washed, dehydrated through a graded Ethanol series, and infiltrated with LR White: Ethanol (1:1, 3:1 and 2 times with 100\% LR White) prior to polymerization in an oven. A standard protocol was used for conventional TEM sample processing [1].

The SEM samples processed by automated processor showed no difference, or were even superior to the conventionally processed ones in microscopic morphology (Fig 1). Suspicious physical damage of fine structure, such as trichomes on the leaves, was not found even though special attention was paid to identifying any alteration caused by mechanical movement of the machine. Automatically processed samples for both conventional TEM and (LR White) iEM were morphologically compatible to those manually processed (Fig. 2).

In conclusion, we believe it is a practical usage of automated tissue processors to prepare plant samples for SEM and TEM, especially with the concern that plant tissues are generally considered difficult to fix and process due to their tendency to float on the surface of liquid reagents during the procedure, whereas tissue processed automatically is held in a basket and completely submerged in the solutions. Programmed agitation helps remove air bubbles on the surface of plant material without applying vacuum. Use of an automated tissue processor eliminates or minimizes experimental variables found in manual processing, such as temperature, agitation, time, and individual handlings. It also minimizes the exposure to hazardous chemicals. This is especially important in the classroom environment with large groups of students by limiting the need for students to handle hazardous chemicals. In addition to freeing their hands, automated tissue processing also allows technicians to better manage their time in a busy multi-task core facility. 


\section{References}

[1] Owen H.A., Makaroff C.A., Protoplasma 185:7-21 1995.

[2] The authors wish to thank Drs. Ljudmilla Timofejeva and Hong Ma for providing Arabidopsis thaliana plants.
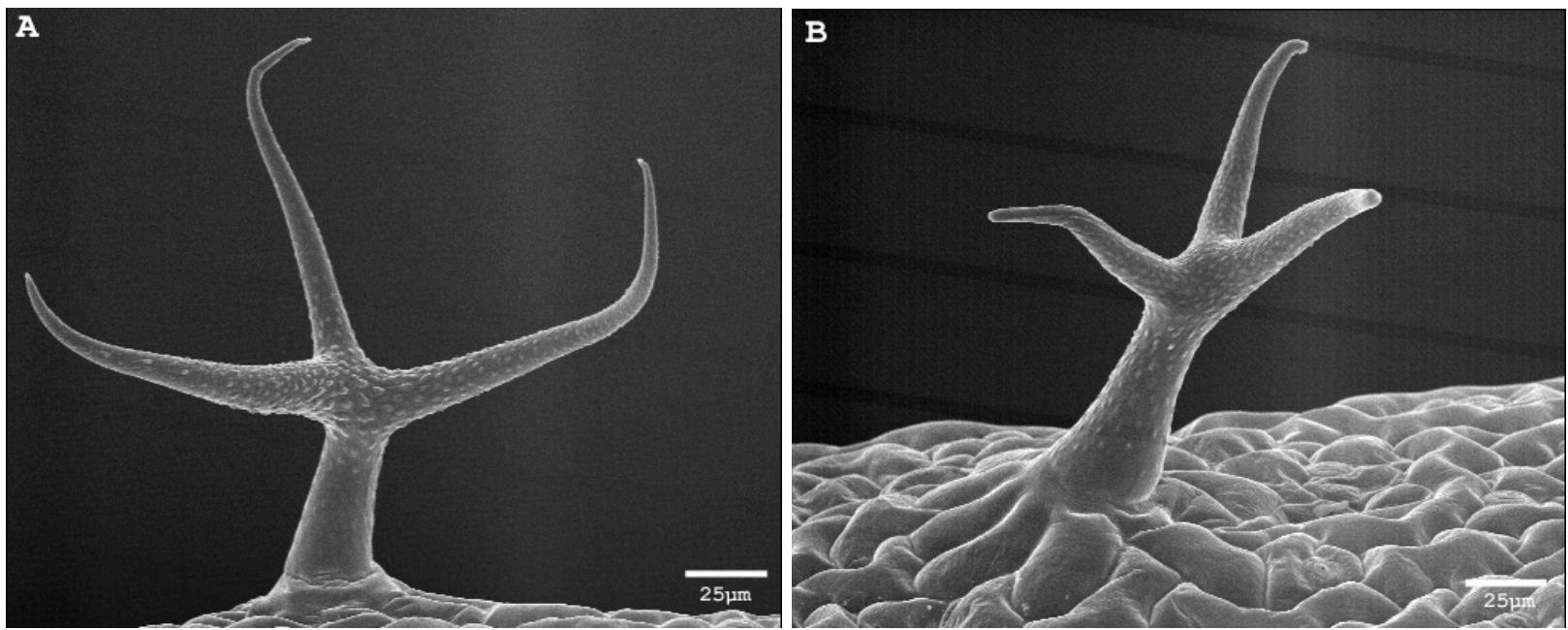

Fig. 1. SEM micrographs of trichomes on the leaves of Arabidopsis thaliana, showing a compatible quality of samples prepared by (A) automated tissue processing and (B) manual processing.
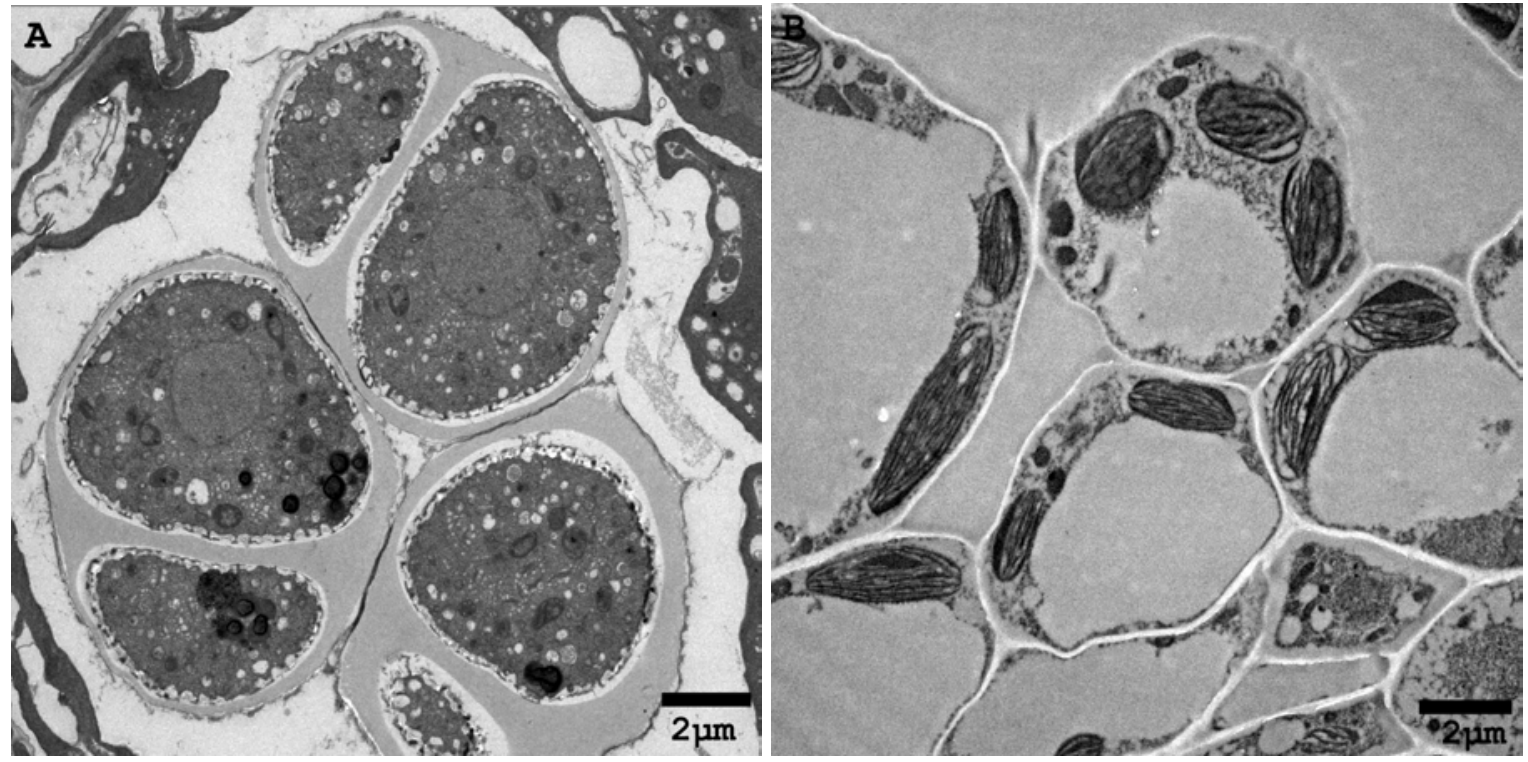

Fig. 2. TEM images of samples of Arabidopsis thaliana floral buds prepared with an automated tissue processor. (A) Conventional TEM samples embedded in Spurr's resin showing microspores are surrounded by a well preserved secreted wall (primexine). (B) Fine structure of epidermal cells embedded in LR White for iEM. 